\title{
Radio Direction-Finding by Transmission and Reception.*
}

\author{
By Dr. R. L. Smith-Rose.
}

$\mathrm{T}$ HE application of the rotating closed loop direction - finder, both as a navigational instrument and as a useful scientific tool in the study of the propagation of electro-magnetic waves, has been developing rapidly during the past few years. So long ago as 1901 the performance of a direction-finder was being studied in America, but owing to its comparative insensitiveness as a receiver the modern radio direction-finder owes its success

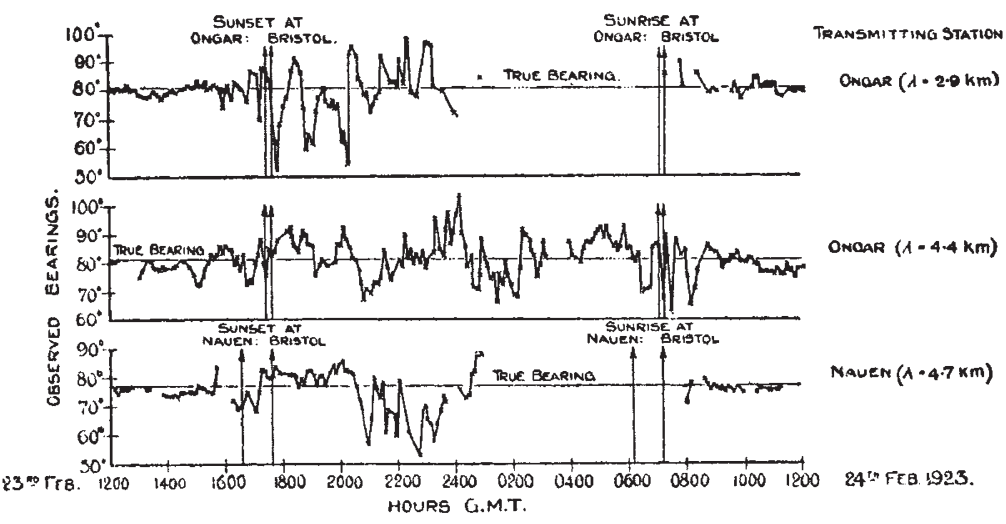

FrG. 1.-Graphs of observed bearings of Ongar (undamped waves, $\lambda=2.9 \mathrm{~km}$. and $4 \cdot 4 \mathrm{~km}$.) and Nauen (undamped waves, $\lambda=4.7 \mathrm{~km}$.) taken at Bristol over a 24 -hour period.

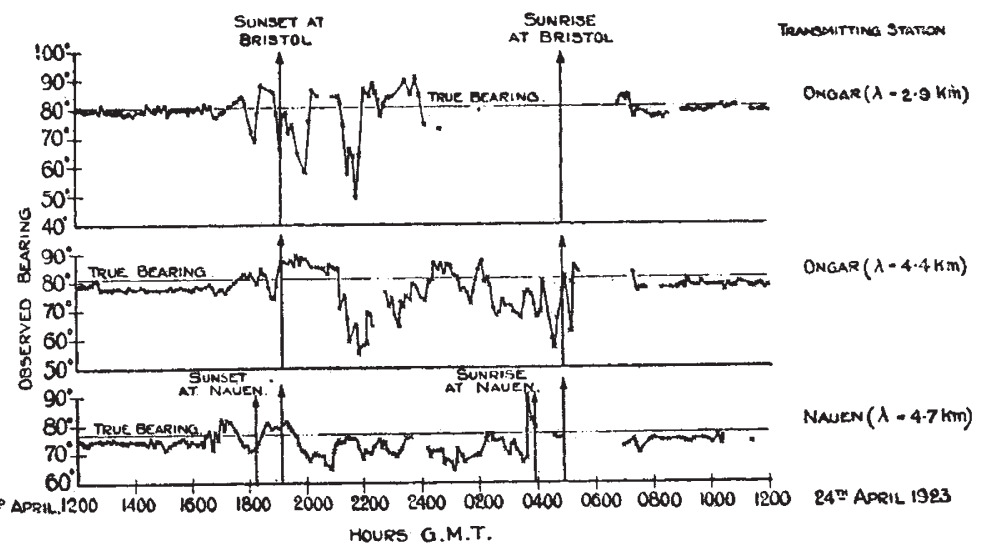

FIG. 2.-Graphs of observed bearings of Ongar (undampea waves, $\lambda=2 \cdot 9 \mathrm{~km}$. and $4 \cdot 4 \mathrm{~km}$.) and Nauen $(\lambda=4 \cdot 7 \mathrm{~km}$.) taken at Bristol over a 24 -hour period.

very largely to the introduction of valve amplifiers, which enabled a moderately large reception range to be obtained. The practical development of the instrument, therefore, dates from about 1915 .

\section{The Radio Direction-Finder.}

The several commercial types of direction-finder now in use employ the same fundamental principle of the reception of vertically polarised $\dagger$ wireless waves by a closed coil. In the attachment of a valve amplifying receiver to the rotating closed

* Abstract of a lecture delivered at the Royal Institution on Feb. 6. ' The term 'vertically polarised' is used here to indicate that the electric force of the wave lies in the vertical plane of propagation. coil, the possibility of obtaining spurious electromotive forces in the system is introduced on account of the phenomena generally known as the 'antenna effect' of the coil and the 'direct pick-up' of signal electromotive force on various parts of the receiver. These spurious electromotive forces make themselves evident by a blurring of the signal zeros, with or without a displacement of these zeros from their correct positions. The methods adopted for overcoming these effects are based upon the use of somewhat elaborate screening arrangements, which were employed in the first place in the construction of direction-finders for accurate research purposes but are now being applied to commercial instruments. In the commercial pattern of rotating loop marine direction-finder, the receiving coil is enclosed in a metallic shielding tube mounted. on a robust column on the deck above the operating room, in which the rotation of the loop and all receiving adjustments can be effected when it is desired to make bearing observations upon any incoming signals. In the case of such use of a direction-finder on board ship, the bearing is taken relative to the ship's head, and it is necessary to obtain this direct from the compass reading in order to make the wireless bearing refer to a great circle direction.

Before the development of valve amplifiers made possible the use of rotatable multi-turn loops, Artom, Bellini, and Tosi suggested and used large frames of a triangular shape for directional wireless communication. The large fixed loops employed in this arrangement, now generally known as the Bellini-Tosi system, are connected to a radiogoniometer, an instrument which reproduces in miniature the directive properties of the external field of the waves. Recent developments of this system for use in ships have resulted in the employment of smaller multi-turn loops which are more conveniently fixed on board and are shielded in a manner similar to the single loops referred to above.

In the application of a direction-finding system to either marine or aerial navigation, it is important to understand clearly the exact conditions under which the observed bearings are accurate, and in other conditions to appreciate the order of magnitude of the possible errors involved and the means of mitigating these where possible. An important factor to be noted from a navigational 
point of view is that of any effect of fog on directionfinding, since it is generally during foggy weather that the majority of marine direction-finders are called into action. The experience of all observers for several years past is in agreement that the existence of fog, whether local or widespread, gives rise to no abnormal effects in directionfinding.

Various conditions may be present in the neighbourhood of a direction-finding station to cause an error in the bearing observed on a distant transmitting station. From various detailed investigations into the causes of such errors, it is known that the most prominent effects are due to masses of metal work and wires, either above or below the earth's surface, and to trees. In most cases where the directional station is situated on land, it is possible to select a site which is largely, if not entirely, immune from such effects. In the case of the use of a direction-finder on board ship, it is impossible to be clear of the metal work of the ship itself, and the resulting bearings are subject to a quadrantal error which can either be compensated by circuit adjustment or corrected for by a chart.

Another type of error which is of some importance in the application of direction-finding to marine navigation is that due to the deviation of wireless waves in crossing a coast line when the path of the waves lies approximately parallel to the coast. On wave-lengths of from 450 metres to 1000 metres, normally used for marine working, the coastal error is of the order of $2^{\circ}-4^{\circ}$ when the direction of transmission is within $20^{\circ}$ of the coast line. The error is always such as to indicate a bending of the waves towards the normal to the coast line in passing from the sea to the land side of the boundary. In connexion with these coastal errors, it is worthy of note that, with the accumulation of experience of the use of directionfinders on board ship, it is now becoming customary to mark out on charts the 'arcs of good bearings' of various transmitting stations within which results of observations are found to be trustworthy.

As an illustration of the reliability of directionfinding as applied to marine navigation, the results of some tests carried out between a directionfinding station on the east coast of England and various ships crossing the North Sea may be quoted. At the various ranges of transmission up to nearly 120 miles, the limiting error of the direction-finder bearing was about $4^{\circ}$. In more than 80 per cent of the cases the wireless bearings were correct to within $1^{\circ}$, while the proportion correct to within $2^{\circ}$ was nearly 95 per cent. These figures refer to bearings taken at a shore station on transmissions from ships across an intervening open sea path at ranges up to about 100 miles. Since it is now generally agreed that an accuracy of $2^{\circ}$ is adequate for most navigation purposes, it is evident that under its ordinary conditions of use the directionfinder is a very trustworthy and useful aid to marine navigation. Similar results to the above are obtainable with the direction-finder installation fitted on board ship and observations made on the transmissions from a shore station. A large number of fixed beacon transmitting stations are now in operation in various parts of the world for the specific purpose of emitting a regular series of signals for the use of ships fitted with directionfinders.

\section{Night Errors.}

When the range of transmission is greater than the figure mentioned above, the accuracy of the observed direction-finder bearings is liable to de-

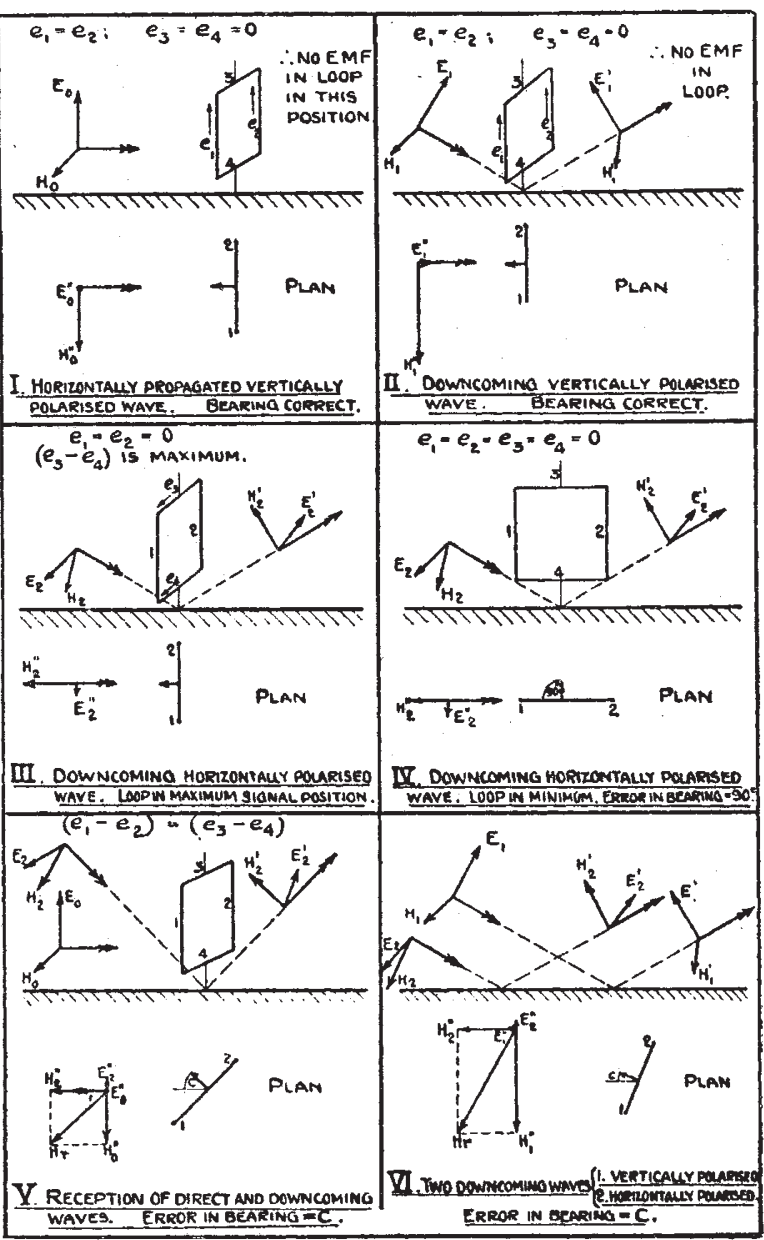

FIG. 3.

preciate during the hours of darkness, when some erratic variations of the observed bearings take place, accompanied by a change in quality of the signal minima. The general nature of these variations can be understood from the typical graphs given in Figs. 1 and 2, which show the apparent bearings of some fixed transmitting stations as observed every few minutes over periods of twentyfour hours at one observing station. On the wavelengths in question, of 2000-4000 metres, it is to be noticed that the day bearings are much steadier in the summer than in the winter months, but that in either case the approach of sunset is accompanied by an increase in the magnitude and frequency of the variable errors which continues

No. 3153, VoL. 125] 
throughout the night until sunrise. Such variable errors may range up to $90^{\circ}$ or more, but it is noteworthy that during the night periods the observed bearings show no signs of a definite systematic error, and so the variations are on the whole equally distributed about the true bearing. The larger errors are also comparatively rare; in fact, observations over long periods have shown that it is seldom that more than 10 per cent of the night observations give an error exceeding $10^{\circ}$.

The cause of the erratic effects and variations observed generally during the night periods is intimately associated with the mode of propagation of electromagnetic waves over the earth's surface. This has been the subject of much detailed research during the past few years, and it is now well known that, in general, two sets of waves travel between a transmitting and receiving station-the direct or ground wave, and the indirect or atmospheric wave. At short distances from the transmitter, the intensity of the indirect wave is generally small, and only the wave transmitted directly along the earth's surface is of importance in producing the received signal. At medium distances, both the direct and the indirect wave are received, while at great distances the major part or the whole of the received signal is due to the indirect wave which has travelled through the upper atmosphere.

In order to explain the variations in apparent bearings on the closed type of direction-finder we have been considering, it is necessary to assume that the plane of polarisation of the downcoming waves may vary. This is undoubtedly caused by a rotation of the plane of polarisation which takes place while the waves are travelling through the ionised regions of the atmosphere and is due to the presence of the earth's magnetic field. In Fig. 3, diagrammatic illustrations are given of a number of simple cases of the reception of wireless waves on a closed loop, and from these it will be seen that it is the horizontal component of the electric force of the downcoming waves which is responsible for producing an error in bearing. So long as the vertically polarised direct wave is strong compared with the horizontally polarised component of the downcoming waves, the error in bearing will be small. Thus the variations in bearings close to the transmitter will be small, a conclusion which is confirmed in practice, for night variations are not experienced at distances less than about 30 miles for overland working.

(To be continued.)

\section{Nobel Prize Awards.}

$\mathrm{F}^{0}$ OLLOWING upon the article in our issue of Mar. 29 , p. 495 , on the history and constitution of the committees of the Nobel Foundation, we print below a complete list of the recipients since 1901--the date of the first distributionof Nobel prizes for physics, chemistry, physiology and medicine, classified according to countries, each country representing the working domicile of the recipient at the time of allotment of the prize.

\section{Physics.}

DENMARK.

1922. Prof. Niels Bohr (Copenhagen), for his services in the investigation of the structure of atoms, and of the radiation emanating from them.

\section{France.}

1903. Prof. Henri Antoine Becquerel (Paris). In recognition of the special services rendered by him in the discovery of spontaneous radioactivity. One half the prize allotted, the other half divided equally between Prof. Pierre Curie (Paris) and Madame Curie, for special services rendered by them in the work they carried out jointly in investigating the phenomena of radiation discovered by Henri Becquerel.

1908. Prof. Gabriel Lippmann (Paris), for his method, based upon the phenomenon of interference, for reproducing colours by photography.

1909. Prof. Ferdinand Braun (Strasbourg), in recognition of services in the development of wireless telegraphy. The prize was divided equally with G. Marconi (Italy).

1920. M. Charles Edouard Guillaume (Sèvres), in recognition of his services to the physics of precision by his discovery of anomalies in nickel steel alioys.

1926. Prof. Jean Perrin (Paris), for his works on the discontinuous structure of matter, and especially for his discovery of the equilibrium of sedimentation.

1929. M. Louis Victor de Broglie (Paris), for his discovery of the wave-character of the electrons.

\section{Germany.}

1901. Prof. Wilhelm Conrad Röntgen (Munich), in recognition of the exceptional services rendered by him in the discovery of the special rays which have been called after him.

1905. Prof. Philipp Lenard (Kiel), for his work in connexion with cathode rays.

1911. Prof. Wilhelm Wien (Würzburg), for his discoveries regarding the laws governing the radiation of heat.

1914. Prof. Max von Laue (Frankfort-on-Main), for his discovery of the defraction of Röntgen rays on passing through crystals.

1918. Prof. Max Planck (Berlin), for his discoveries in connexion with the quantum theory. Awarded in 1919.

1919. Prof. Johannes Stark (Greifswald), for his discovery of the Doppler effect in canal rays, and of the decomposition of spectrum lines in an electric field.

1921. Prof. Albert Einstein (Berlin), for his services to the theory of physics, and especially for his discovery of the law of the photo-electric effect.

1925. Prof. James Franck (Göttingen), and Prof. Gustav Hertz (Halle), for their discovery of the laws governing the impact of an electron upon an atom. The prize was divided equally in 1926.

No. 3153, Vor. 125] 